\title{
Episodes discussed in detail
}

American Gothic. Created by Shaun Cassidy. Perf. Gary Cole, Lucas Black and Paige Turco. CBS, 1995-1996

1.1 “Pilot.” Wr. Shaun Cassidy. Dir. Peter O’Fallon. September 22, 1995.

1.2 “A Tree Grows in Trinity.” Wr. Shaun Cassidy. Dir. Michael Katleman. September 29, 1995.

1.3 "Eye of the Beholder." Wr. Shaun Cassidy and Judi Ann Mason. Dir. Jim Charleston. October 6, 1995.

1.4 “Damned If You Don't." Wr. Stephen Gaghan and Michael R. Perry. Dir. Lou Antonio. October 10, 1995.

1.5 "Dead to the World." Wr. Robin Green, Mitchell Burgess, Shaun Cassidy, Michael R. Perry and Stephen Gaghan. Dir. James A. Contner. October 13, 1995.

1.10 "Inhumanitas." Wr. Stephen Gaghan and Michael R. Perry. Dir. Bruce Seth Green. January 17, 1996.

1.11 "The Plague Sower." Wr. Robert Palm. Dir. Mel Damski. January 24, 1996.

1.17 “The Buck Stops Here." Wr. Steve De Jarnatt. Dir. Lou Antonio. July 10, 1996.

1.22 "Strangler." Wr. Stephen Gaghan and Michael R. Perry. Dir. Doug Lefler. Unaired. 


\section{American Horror Story. Created by Brad Falchuk and Ryan Murphy. Perf. Jessica Lange and Evan Peters. FX, 2011-}

1.1 "Pilot." Wr. Brad Falchuk and Ryan Murphy. Dir. Ryan Murphy. October 5, 2011.

1.3 "Murder House." Wr. Jennifer Salt. Dir. Bradley Buecker. October 19, 2011.

1.4 "Halloween Part 1." Wr. James Wong. Dir. David Semel. October 26, 2011.

1.5 "Halloween Part 2." Wr. Tim Minear. Dir. David Semel. November 2, 2011.

1.6 “Piggy, Piggy.” Wr. Jessica Sharzer. Dir. Michael Uppendahl. November 9, 2011.

1.7 “Open House.” Wr. Brad Falchuk. Dir. Tim Hunter. November 16, 2011.

1.8 “The Rubber Man.” Wr. Ryan Murphy. Dir. Miguel Arteta. November 23, 2011.

1.9 “Spooky Little Girl.” Wr. Jennifer Salt. Dir. John Scott. November 30, 2011.

1.11 "Birth." Wr. Tim Minear. Dir. Alfonso Gomez-Rejon. December 14, 2011.

1.12 "Afterbirth." Wr. Jessica Sharzer. Dir. Bradley Buecker. December 21, 2011.

Angel. Created by Joss Whedon and David Greenwalt. Perf. David Boreanaz. WB, 1999-2004

1.14 "I've Got You Under My Skin." Wr. Jeannine Renshaw and David Greenwalt. Dir. R. D. Price. February 15, 2000.

1.15 "The Prodigal." Wr. Tim Minear. Dir. Bruce Seth Green. February 22, 2000.

1.20 "War Zone.” Wr. Garry Campbell. Dir. David Straiton. May 9, 2000.

2.6 "Guise will be Guise." Wr. Jane Espenson. Dir. Krishna Rao. November 7, 2000.

2.8 "The Shroud of Rahmon.” Wr. Jim Kouf. Dir. David Grossman. November 21, 2000.

2.10 "Reunion." Wr. Tim Minear and Shawn Ryan. Dir. James A. Contner. December 19, 2000.

2.19 “Belonging.” Wr. Shawn Ryan. Dir. Turi Meyer. May 1, 2001. 
2.20 "Over the Rainbow.” Wr. Mere Smith. Dir. Fred Keller. May 8, 2001.

2.21 “Through the Looking Glass.” Wr. and dir. Tim Minear. May $15,2001$.

3.6 "Billy." Wr. Tim Minear and Jeffrey Bell. Dir. David Grossman. October 29, 2001.

3.20 “A New World.” Wr. Jeffrey Bell. Dir. Tim Minear. May 6, 2002.

4.11 "Soulless." Wr. Sarah Fain and Elizabeth Craft. Dir. Sean Astin. February 5, 2003.

4.20 "Sacrifice." Wr. Ben Edlund. Dir. David Straiton. April 23, 2003.

5.5 "Life of the Party." Wr. Ben Edlund. Dir. Bill Norton. October 29, 2003.

5.7 “Lineage." Wr. Drew Goddard. Dir. Jefferson Kibbee. November 12, 2003.

Bewitched. Created by Sol Saks. Perf. Elizabeth Montgomery. ABC, 1964-1972

1.3 “It Shouldn't Happen to a Dog.” Wr. Jerry Davis. Dir. William Asher. October 1, 1964.

2.2 “A Very Special Delivery.” Wr. Howard Leeds. Dir. William Asher. September 23, 1965.

Buffy the Vampire Slayer. Created by Joss Whedon. Perf. Sara Michelle Gellar. WB and UPN, 1997-2003

5.1 "Buffy vs. Dracula." Wr. Marti Noxon. Dir. David Solomon. Original air date September 26, 2000.

Carnivàle. Created by Daniel Knauf. Perf. Nick Stahl, Michael J. Anderson, and Clea DuVall. HBO, 2003, 2005

1.1 “Milfay.” Wr. Daniel Knauf. Dir. Rodrigo García. September 14, 2003.

1.6 "Pick a Number.” Wr. Ronald D. Moore. Dir. Rodrigo García. October 19, 2003.

1.11 "Day of the Dead." Wr. Toni Graphia. Dir. John Patterson. November 23, 2003. 
1.12 "The Day That Was the Day." Wr. Ronald D. Moore. Dir. Rodrigo García. November 30, 2003.

2.4 “Old Cherry Blossom Road." Wr. Dawn Prestwich and Nicole Yorkin. Dir. Steve Shill. January 30, 2005.

2.7 "Damascus, NE." Wr. John J. McLaughlin and William Schmidt. Dir. Alan Taylor. February 20, 2005.

Kolchak, the Night Stalker. Created by Jeffrey Grant Rice. Perf. Darren McGavin. ABC, 1974-1975

1.2 "The Zombie." Wr. Zekial Marko and David Chase. Dir. Alexander Grasshoff. September 20, 1974.

\section{Millennium. Created by Chris Carter. Perf. Lance Henriksen and} Megan Gallagher. Fox, 1996-1999

1.1 "Pilot." Wr. Chris Carter. Dir. David Nutter. October 25, 1996.

1.8 “The Well-Worn Lock.” Wr. Chris Carter. Dir. Ralph Hemecker. December 20, 1996.

1.16 “Covenant.” Wr. Robert Moresco. Dir. Rod Pridy. March 21, 1997.

1.18 "Lamentation.” Wr. Chris Carter. Dir. Winrich Kolbe. April 18, 1997.

2.1 "The Beginning and the End." Wr. Glen Morgan and James Wong. Dir. Thomas J. Wright. September 19, 1997.

2.2 "Beware of the Dog." Wr. Glen Morgan and James Wong. Dir. Allen Coulter. September 26, 1997.

2.6 "The Curse of Frank Black." Wr. Glen Morgan and James Wong. Dir. Ralph Hemecker. October 31, 1997.

2.19 “Anamnesis." Wr. Erin Maher and Kay Reindl. Dir. John Peter Kousakis. April 17, 1998.

2.20 “A Room with No View." Wr. Ken Horton. Dir. Thomas J. Wright. April 24, 1998.

2.21 "Somehow, Satan Got Behind Me." Wr. Darin Morgan. Dir. Darin Morgan. May 1, 1998.

2.22 “The Fourth Horseman.” Wr. Glen Morgan and James Wong. Dir. Dwight H. Little. May 8, 1998.

2.23 “The Time Is Now." Wr. Glen Morgan and James Wong. Dir. Thomas J. Wright. May 15, 1998. 
3.5 “...Thirteen Years Later.” Wr. Michael R. Perry. Dir. Thomas J. Wright. October 30, 1998.

3.13 "Antipas." Wr. Chris Carter and Frank Spotnitz. Dir. Thomas J. Wright. February 12, 1999.

\section{Point Pleasant. Created by Marti Noxon. Perf. Elisabeth Harnois} and Grant Show. Fox, 2005-2006

1.3 “Who's Your Daddy?” Wr. Ben Edlund. Dir. Marita Grabiak. January 27, 2005.

1.8 "Swimming with Boyd." Wr. Jenny Lynn and Jeff Diaz. Dir. James Contner. March 17, 2005.

1.9 "Waking the Dead." Wr. Robert Doherty, Ben Edlund, and Zack Estrin. Dir. Marita Grabiak. June 19, 2005.

1.10 "Hell Hath No Fury Like a Woman Choked." Wr. Ron Milbauer, Terri Hughes Burton, and Jeff Diaz. Dir. Mel Damski. June 26, 2005.

1.11 "Missing." Wr. Andrea Newman and P. K. Simonds. Dir. Michael Lange. July 3, 2005.

1.12 "Mother's Day.” Wr. Jeff Diaz, Robert Doherty, Zack Estrin, and Diego Gutierrez. Dir. Felix Alcala. September 9, 2006.

1.13 "Let the War Commence.” Wr. Jenny Lynn and Marti Noxon. Dir. Chris Long. September 9, 2006.

Supernatural. Created by Eric Kripke. Perf. Jensen Ackles and Jared Padalecki. WB and CW, 2005-

1.6 “Skin.” Wr. John Shiban. Dir. Robert Duncan McNeill. October 18, 2005.

1.8 “Bugs." Wr. Rachel Nave and Bill Coakley. Dir. Kim Manners. November 8, 2005.

1.9 "Home." Wr. Eric Kripke. Dir. Ken Girotti. November 15, 2005.

1.10 "Asylum." Wr. Richard Hatem. Dir. Guy Norman Bee. November 22, 2005.

1.11 "Scarecrow." Wr. John Shiban and Patrick Sean Smith. Dir. Kim Manners. January 10, 2006.

1.12 "Faith." Wr. Sera Gamble and Raelle Tucker. Dir. Allan Kroeker. January 17, 2006. 
1.14 “Nightmare.” Wr. Sera Gamble and Raelle Tucker. Dir. Phil Sgriccia. February 7, 2006.

1.18 "Something Wicked.” Wr. Daniel Knauf. Dir. Whitney Ransick. April 6, 2006.

1.20 "Dead Man's Blood." Wr. Cathryn Humphris and John Shiban. Dir. Tony Whamby. April 20, 2006.

2.7 "The Usual Suspects." Wr. Cathryn Humphris. Dir. Mike Rohl. November 9, 2006.

2.9 “Croatoan.” Wr. John Shiban. Dir. Robert Singer. December 7, 2006.

2.10 “Hunted.” Wr. Raelle Tucker. Dir. Rachel Talalay. January 11, 2007.

2.11 "Playthings." Wr. Matt Witten. Dir. Charles Beeson. January 18, 2007.

2.12 “Nightshifter.” Wr. Ben Edlund. Dir. Phil Sgriccia. January 25, 2007.

2.13 "Houses of the Holy." Wr. Sera Gamble. Dir. Kim Manners. February 1, 2007.

2.19 "Fulsom Prison Blues." Wr. John Shiban. Dir. Mike Rohl. April 26, 2007.

2.22 “All Hell Breaks Loose 2.” Wr. Eric Kripke and Michael T. Moore. Dir. Robert Singer. May 17, 2007.

4.13 "After School Special." Wr. Andrew Dabb and Daniel Loflin. Dir. Adam Kane. January 29, 2009.

4.14 "Sex and Violence." Wr. Cathryn Humphris. Dir. Charles Beeson. February 5, 2009.

4.18 "The Monster at the End of this Book." Wr. Julie Siege and Nancy Weiner. Dir. Mike Rohl. April 2, 2009.

4.21 "When the Levee Breaks." Wr. Sera Gamble. Dir. Robert Singer. May 7, 2009.

5.13 "The Song Remains the Same.” Wr. Sera Gamble and Nancy Weiner. Dir. Steve Boyum. February 4, 2010.

6.15 "The French Mistake.” Wr. Ben Edlund. Dir. Charles Beeson. February 25, 2011. 